Presented at Fall Meeting of the Materials Reseach Society, Boston, MA, December 1-5, 1997; to be published in Proceedings

$$
\operatorname{CONF}-97 / 20 /--
$$

\title{
DIRECT OBSERVATIONS OF ATOMIC STRUCTURES OF DEFECTS IN GaN BY HIGH-RESOLUTION Z-CONTRAST STEM
}

Y. Xin, ${ }^{1,2}$ S. J. Pennycook, ${ }^{1}$ N. D. Browning, ${ }^{2}$ P. D. Nellist, ${ }^{3}$

S. Sivananthan, ${ }^{2}$ J.-P. Faurie, ${ }^{4}$ and P. Gilbart ${ }^{4}$

${ }^{1}$ Solid State Division, Oak Ridge National Laboratory

P.O. Box 2008, Oak Ridge, Tennessee 37831-6030

2Department of Physics, University of Illinois at Chicago

Chicago, Illinois 60607-7059

RECEIVED

JAH 261998

0.91

${ }^{3}$ Cavendish Laboratory, University of Cambridge

Madingley Road, Cambridge CB3 OHE, United Kingdom

${ }^{4}$ CRHEA-CNRS, rue Bernard Gregory

06560 Valbonne, France

\footnotetext{
"The submitted manuscript has been authored by a contractor of the U.S. Government under contract No. DE-AC05-960R22464. Accordingly, the U.S. Government retains a nonexclusive, royalty-free license to publish or

reproduce the published form of this

contribution, or allow others to do so, for U.S.

Government purposes."
}

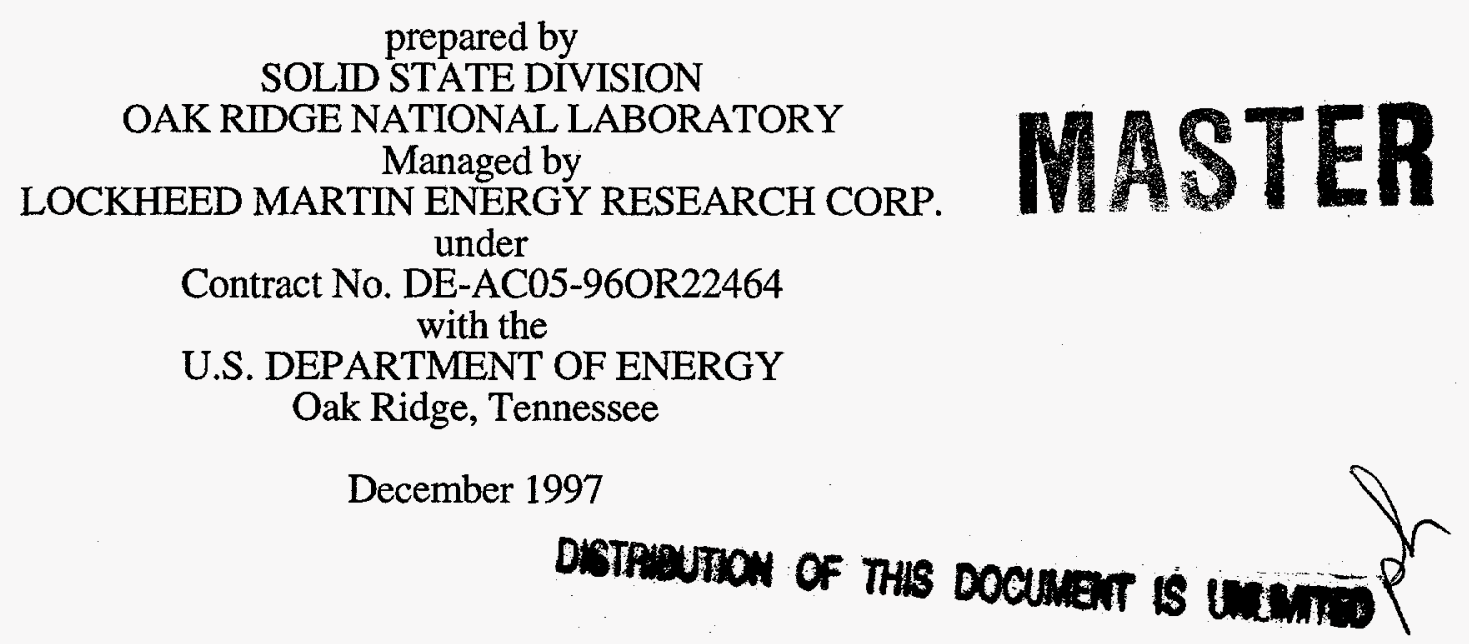




\section{DISCLAIMER}

This report was prepared as an account of work sponsored by an agency of the United States Goverament. Neither the United States Government nor any agency thereof, nor any of their employees, makes any warranty, express or implied, or assumes any legal liability or responsibility for the accuracy, completeness, or usefulness of any information, apparatus, product, or process disclosed, or represents that its use would not infringe privately owned rights. Reference herein to any specific commercial product, process, or service by trade name, trademark, manufacturer, or otherwise does not necessarily constitute or imply its endorsement, recommendation, or favoring by the United States Government or any agency thereof. The views and opinions of authors expressed herein do not necessarily state or reflect those of the United States Government or any agency thereof. 


\section{DISCLAIMER}

Portions of this document may be illegible electronic image products. Images are produced from the best available original document. 
Y. Xin 1.2, S.J. Pennycook ${ }^{2}$, N.D. Browning ${ }^{1}$, P. D. Nellist ${ }^{3}$, S. Sivananthan', J-P Faurie ${ }^{1,4}$ and P. Gibart ${ }^{4}$

'Department of Physics, University of Illinois at Chicago, Chicago. IL 60607-7059, USA.

${ }^{2}$ Solid State Division, Oak Ridge National Laboratory, Oak Ridge, TN 37381-6031, USA

${ }^{3}$ Cavendish Laboratory, Cambridge University,Madingley Road,Cambridge CB3 OHE,UK

${ }^{4}$ CRHEA-CNRS, rue Bernard Gregory, 06560 Valbonne, France.

\section{ABSTRACT}

$\mathrm{GaN} /(0001)$ Sapphire grown by low pressure MOVPE is studied by high resolution Z-contrast imaging using STEM. First direct observation of the threading dislocation with edge character shows the atomic core structure, which appears to have a similar configuration to the $110-10\}$ surface. The surfaces of the nanopipe walls are on $\{10-10\}$ with the terminating layer between the atoms with one bond per pair. In addition, the high resolution Z-contrast image of the prismatic stacking fault confirms the results by conventional HRTEM.

\section{INTRODUCTION}

Since the success in the production of high efficiency light emitting diodes (LED) based on gallium nitride material[1], wide gap nitride semiconductors have attracted much attention recently due to their promising performance in short-wave length LED, blue lasers, and other wide range of electronic-opto applications. Many efforts therefore have been mainly devoted into two parts, one on the improvement of the crystal quality of the nitride layers by different growth methods, the other on the investigation of the less-known electronic and optical properties of these materials by various methods and also by theoretical calculations.

The most interesting and intriguing issue concerning $\mathrm{GaN}$ lies in the fact that the GaN-based LED is found to be remarkably insensitive to the high density of structural defects such as dislocations in the films, which is most unusual compared with LEDs based on III-V arsenides and phosphides[2]. It is well know that dislocations introduce non-radiative recombination centres in these GaAsP based LED.

In this paper, we report the first direct observation of core structures of threading dislocations with edge character in hexagonal $\mathrm{GaN}$, and some other defects, such as prismatic stacking fault, nanopipe. . These observations allow the reasons for the insensitivity to defects to be intimated.

\section{EXPERIMENT}

The GaN films used in this study were grown by metalorganic vapor phase epitaxy (MOVPE), which have been reported in detail in other papers [3]. A brief description of the growth process and general features of the film is presented here. The films were deposited on (0001) sapphire substrate in a low-pressure MOVPE reactor. The substrate was nitridated at high temperature, and the buffer layer grown at $525^{\circ} \mathrm{C}$, followed by heat ramping up to $1150^{\circ} \mathrm{C}$, and growth proceeded under hydrogen with V/III ratio 1400 .

Conventional TEM images were obtained using Philips CM12 microscope, while the atomic structures of the defects are studied by high resolution Z-contrast imaging [4] on a $300 \mathrm{kV}$ scanning transmission electron microscope (STEM) VG $\mathrm{HB} 603$ with a resolution of $1.3 \AA$. High resolution Z-contrast imaging is a unique technique different from conventional high resolution imaging. It is an incoherent imaging technique, in which atomic columns are imaged with a brightness proportional to mean square atomic number (Z). Atomic column positions can therefore be accurately determined. It provides intuitive and direct information which is most useful in the study of defect structures in semiconductor materials[9]. In an attempt to detect localised electronic 
states around the individual dislocation in $\mathrm{GaN}$, atomic resolution electron energy loss spectroscopy (EELS) were carried out using a VG STEM 501 with spacial resolution of $2.2 \AA$.

\section{RESULTS AND DISCUSSION}

\section{General Microstructure}

The dislocation density of the threading dislocations in these films is around $10^{10} \mathrm{~cm}^{-2}$, the majority being of edge character, as determined by the conventional TEM study shown in Fig.1 Fig. $1 \mathrm{a}$ is a weak beam dark field image of a cross sectional sample using reflection $\mathrm{g}=0002$, in which pure screw or mixed dislocation with screw character are in contrast, while Fig.1b is an image of the same region using $g=1-100$, in which dislocations with edge character are shown. Fig. $2 \mathrm{a}$ is an annular dark field image at low magnification showing that there are nanocavities in the $\mathrm{GaN}$ buffer layer region. The buffer layer contains planar defects, such as stacking faults and thin layers of cubic intergrowth. In Fig.2c, the image is down [11-20] showing only Ga atoms of the epilayer. The GaN epilayer above the substrate surface at the interface appears to be atomicly abrupt.

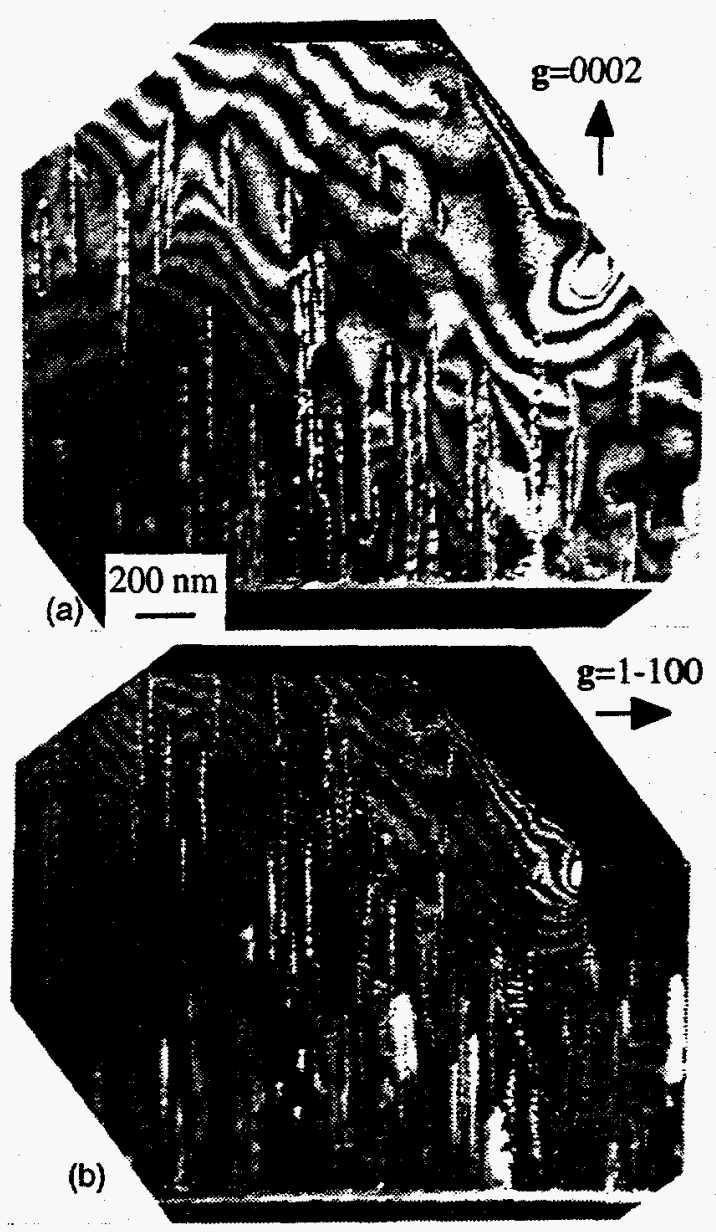

Fig.1 Weak beam dark field images of a cross sectional sample. (a) screw and mixed type dislocations, (b) Edge dislocations and some mixed ones.
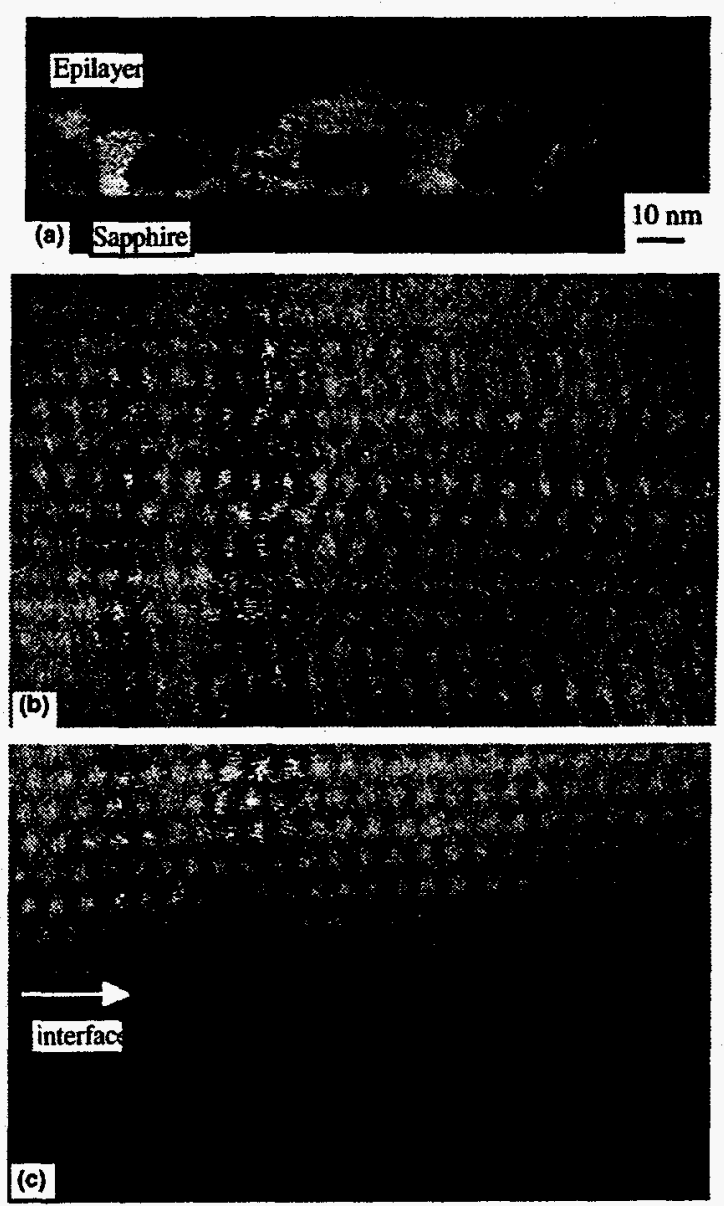

Fig.2 STEM annular dark field images of the crosssectional sample. (a) At low magnification; (b)High resolution image of the buffer layer; (c) Interface between $\mathrm{GaN}$ and sapphire substrate (arrowed).(The distance between atomic planes is $2.6 \AA$.) 
Fig3a is an as-required high resolution Z-contrast image looking down the c-axis of a threading dislocation. Each bright spot is an atomic column of alternating $\mathrm{Ga}$ and $\mathrm{N}$ atoms. The dislocation has an edge component of the Burgers vector of $\mathbf{b}=1 / 3[11-20]$. Fig.3b is a maximum entropy [9] image of Fig.3a, in which each individual atomic column shown as small white dot. It is retrieved from the original image and represented by the object convoluted with a small Gaussian probe. The reconstruction of the 'most likely' object is consistent with the experimental image.
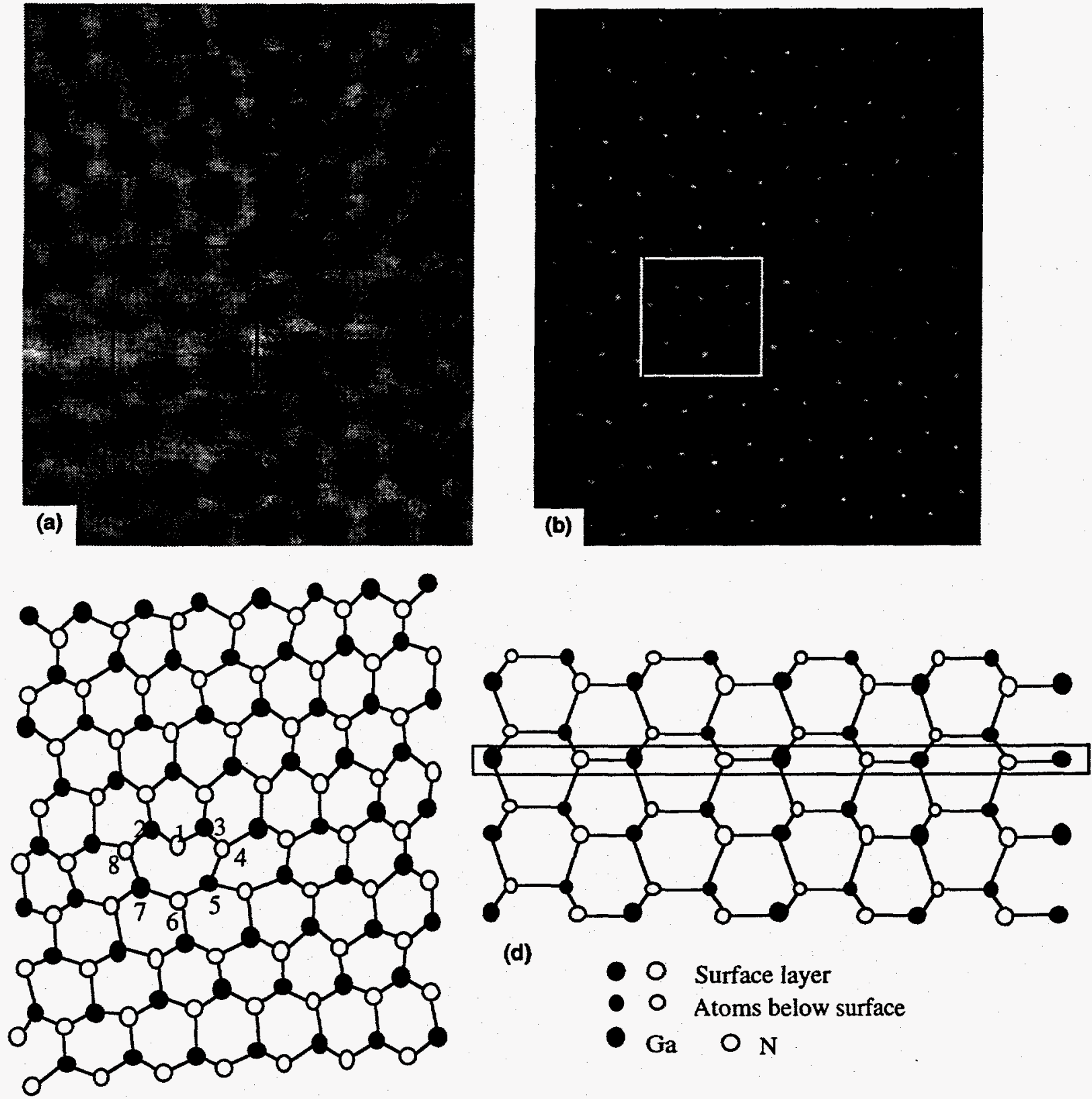

(d)

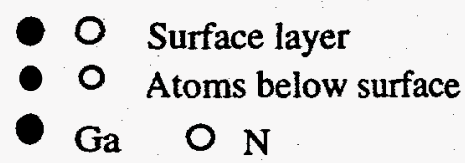

(c)

Fig.3 High resolution Z-contrast image of an edge threading dislocation down [0001]. (a) Asrequired image with the core in the boxed region; (b) Maximum entropy image of the raw data; (c) Model of the core structure of the dislocation with one double Ga-N layer projected onto (0001) plane; (d)top view of $\{10-10\}$ surface, in which one line of the dimmers is drawn in a box indicating the side view of the atomic configuration of the core.(The distance between atomic columns is $1.84 \AA$.) 
The core is just a very neat 8 -fold ring, indicated by numbers 1 to 8 in the schematic( Fig.3c). Comparing the core observed here with the configuration of the $\{10-10\}$ surface[10], it is found that the core has the same configuration of the $\{10-10\}$ surface, and it is just composed of one line of the dimmers of that surface layer, as shown in the boxed region of Fig.3d. Comparing the calculation by Northrup of the $\{10-10\}$ surface, it is believed that the core would have similar relaxation mechanism by the contraction of the original bond between $\mathrm{Ga}$ and $\mathrm{N}$ as well as by rehybridization of each species along the core column. Relaxation would also possibly occur of the core column by small contraction to the bonded atoms in the basal plane, i.e. column 1 is closer to column 2 and column 3 , which in fact is evidenced by the image. We might therefore expect the core behaves the same as the $\{10-10\}$ surface, i.e. there are no deep defect states caused by edge threading dislocations in $\mathrm{GaN}$. More detailed study of the core structure of the edge type threading dislocations could be found in our other paper [12]. Indeed, the above suggestions are confirmed by recently theoretical work on dislocations in GaN[11].

$\mathrm{N}$ K-edges of EELS spectrum on and off dislocations were compared and no difference or pre-edge features were found, which is consistent with the core structure observation.

\section{Nanopipes}

Fig. 4 is the high resolution Z-contrast image of the edges of a nanopipe looking down the [0001] direction. The bright dots in the images show the columns of mixed $\mathrm{Ga}$ and $\mathrm{N}$.

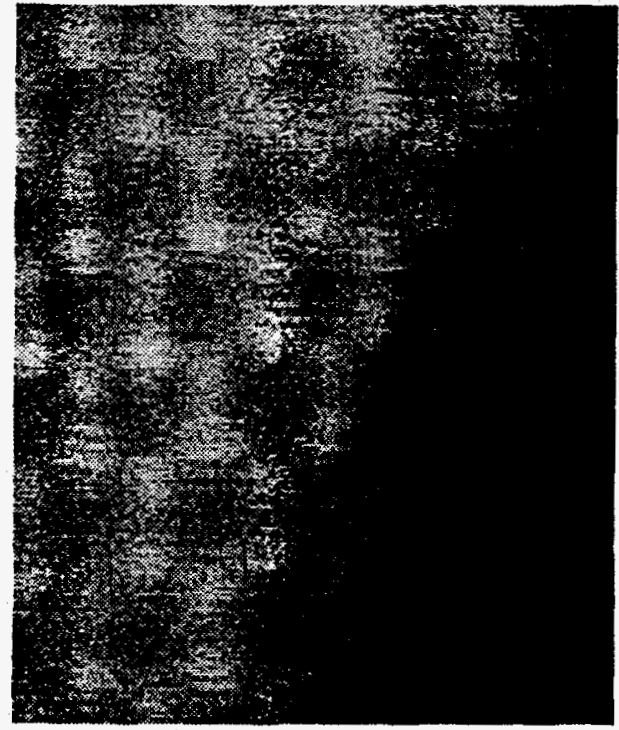

(a)

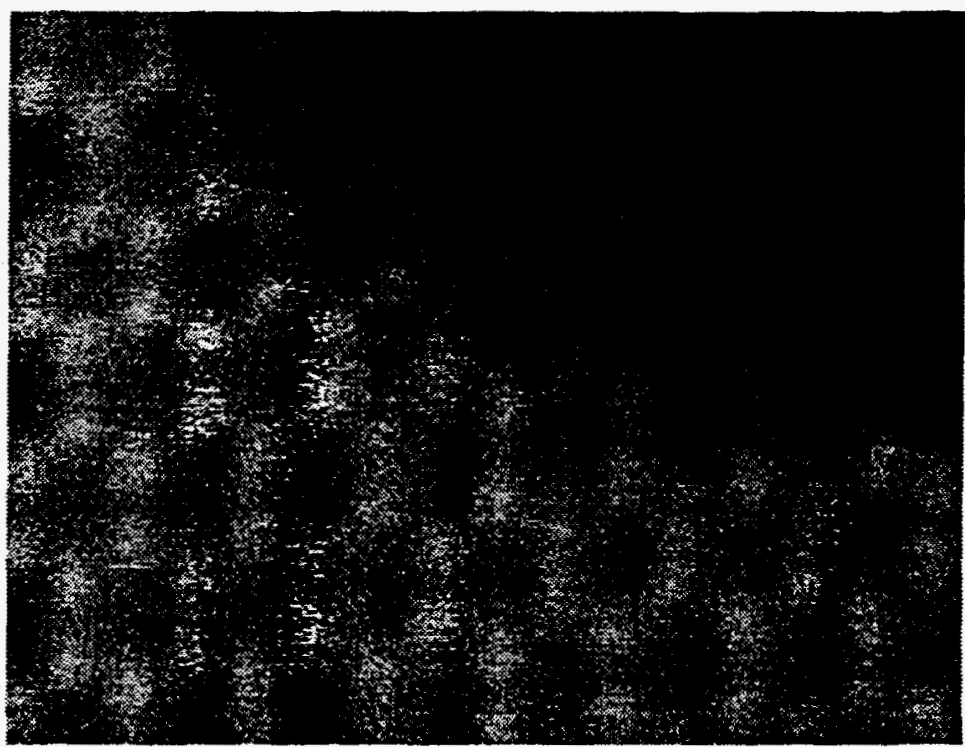

(b)

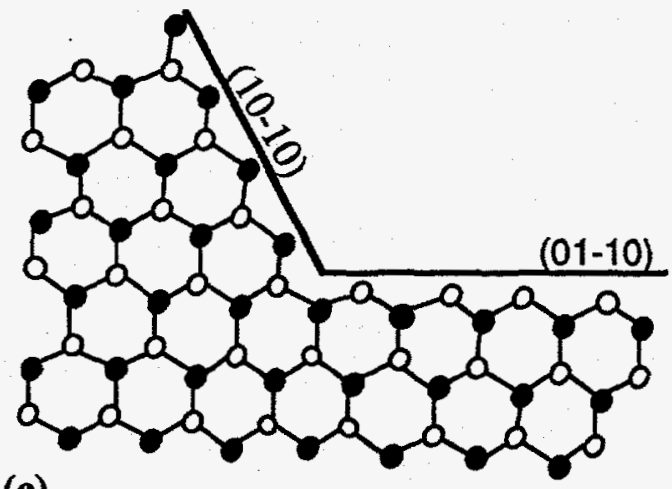

(c)
Fig.4 High resolution Z-contrast images of the edges of the nanopipes.

(a) one of the straight edge;

(b) the corner joining the two straight edges;

(c) sketch of one double $\mathrm{Ga}-\mathrm{N}$ layer projected onto $(0001)$ showing the terminating surfaces. 
The terminating of the $\{10-10\}$ surface is between the atom pair with one bond as shown in the sketch. From Fig.4 a, it seems that the edge has sharp steps from thick to thinner region rather than a gradually decreased thickness.

\section{Prismatic stacking fault}

Planar defects in GaN such as stacking-mismatch boundaries [5] and inversion domain boundaries [6] have been well studied. One type is the prismatic stacking fault which could exist as short segment in the sample as well as in the form of domain boundaries [7]. It is already known that the displacement across the boundary is $1 / 2<1-101>$ [7]. The high resolution Z-contrast image shown in Fig.5 of the stacking fault looking down [0001] confirms the previous study and gives the intuitive structure of the fault without any need of simulation. It is composed of four- and eight-fold rings along the fault (Fig. 5a). The fault ends in the perfect crystal region by a dislocation of a seven-fold ring with edge and screw character (Fig.5b). By simple consideration of bond length and bond angles, it could be seen from the images that the bonds across the fault have similar lengths compared to the bond length in the perfect region. However, the bond angles are significantly distorted compared to the $109^{\circ}$ of perfect crystal. Although no dangling bonds are assumed from the structure of the fault, it is considered here that the prismatic stacking fault could have deep defect state in the band gap because of the distorted bond angles. Indeed, EELS expriment from these faults has detected defect states in the gap [8].
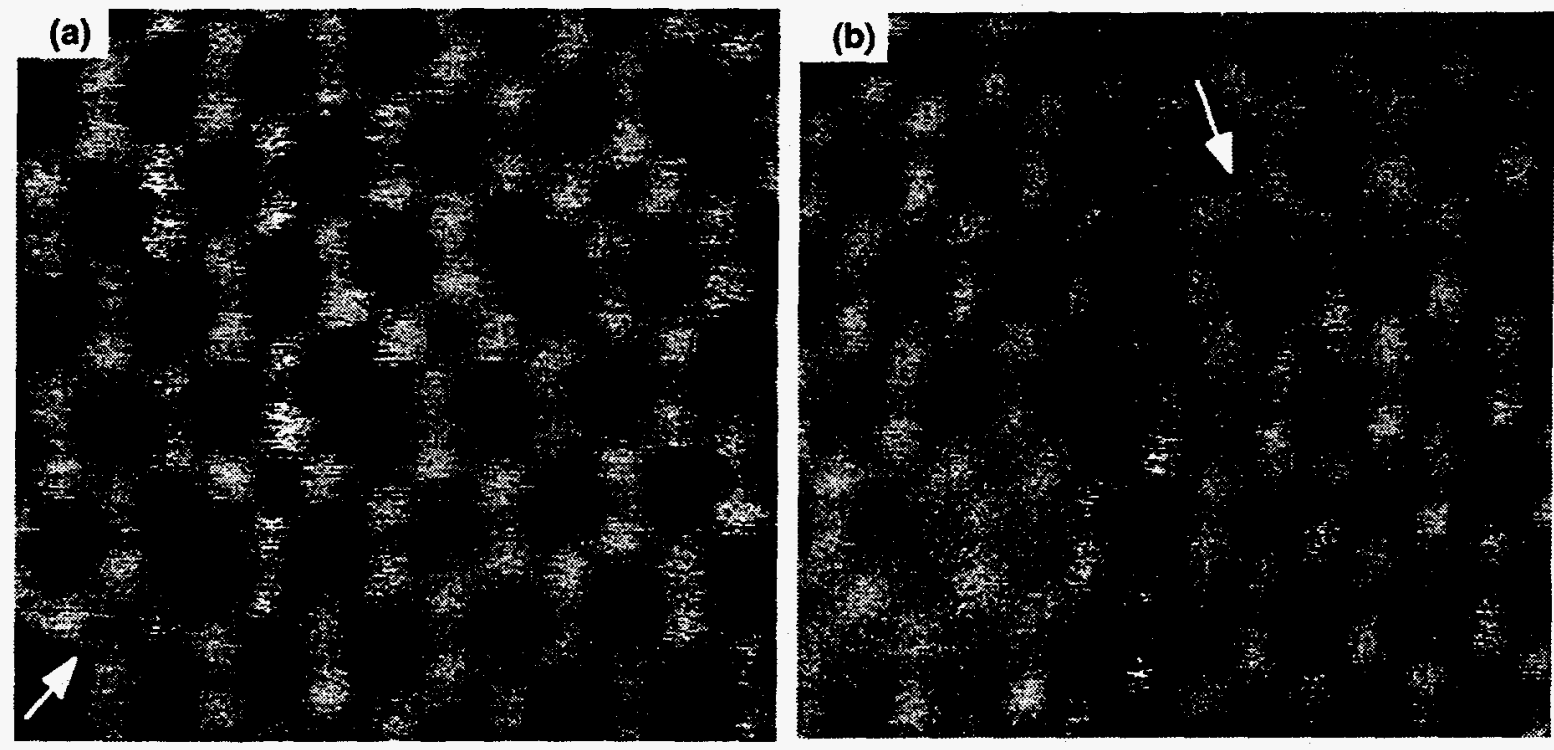

Fig.5 High resolution Z-contrast images down [0001] of the prismatic stacking fault with $\mathbf{R}=1 / 2<1-101>$, (a) a prismatic stacking fault indicated by an arrow; (b) the fault bounded by a dislocation of a seven-fold ring, indicated by an arrow.

\section{CONCLUSIONS}

In summary, first direct observation of atomic core structures of threading dislocations in $\mathrm{GaN}$ is presented. The core of the dislocation with edge character is seen to contain an alternating chain of $\mathrm{Ga}$ and $\mathrm{N}$ which are presumed to relax to avoid dangling bonds giving a charge neutral structure similar to the $\{10-10\}$ surface. It also confirms the proposed model by the theoretical calculations of threading dislocations. Terminating surfaces of the nanopipes and prismatic stacking faults are also determined. 


\section{ACKNOWLEDGMENTS}

This research was sponsored by the Division of Materials Sciences, USDOE, under contract DEAC05-960R22464 with Lockheed Martin Energy Research Corporation, in part by DOE under grant number DOE 96ER45610. Metal and ceramic Division in ORNL is acknowledged for the permission for using CM12 microscope.

\section{REFERENCES}

1. S. Nakamura, T. Mukai and M. Senoh, Jpn. J. Aool. Phys. 30, p. L1998 (1991).

2. S.D. Lester, F.A. Ponce, M.G. Craford, and D.A. Steigerwald, Appl. Phys. Lett.,66, p. 1249 (1995).

3. P. Vennegues, B. Beaumont, M. Vaille and P. Gibart, J. Crystal Growth, 173, p. 249 (1997).

4. S.J. Pennycook, N.D. Browning, D.E. Jessen, M.F.Chisholm and A.J. McGibbon, Appl. Phys. A57, p, 385 (1993).

5. B.N. Serdlov, G.A. Martin, H. Morkoc and D.J. Smith, Appl. Phys. Lett., 67, p. 2063 (1995)

6. L.T. Romano, J.E.Northrup, M.A.O'Keefe, Appl. Phys. Lett., 69, p. 2394 (1996).

7. Y. Xin, P.D. Brown, C.J. Humphreys, T.S. Cheng and C.T. Foxon, Appl. Phys. Lett., 70, p. 1308 (1997).

8. M.K.H. Natusch, G. Botton, R. Broom, P.D. Brown, D. Tricker and C.J. Humphreys, this proceeding.

9. A.J. McGibbon, S.J. Pennycook and J.E. Angelo, Science, 269, p. 519 (1995).

10. J.E. Northrup and J. Neugebauer, Phys. Rev. B, 53, p. R10477 (1996).

11. J. Elsner, R. Jones, P.K. Sitch, V.D. Porezag, M. Elstner, Th. Frauenheim, M.I. Heggie, S. Oberg and P.R. Briddon, Phys. Rev. Lett., 79, p. 3672 (1997).

12. Y. Xin, S.J. Pennycook, N.D. Browning, P. D. Nellist, S. Sivananthan, J-P Faurie and P. Gibart, submitted to Appl. Phys. Lett. 